\title{
Multilevel, Room-Temperature Nanoimprint Lithography for Conjugated Polymer-Based Photonics
}

2005

Vol. 5, No. 10

1915-1919

\author{
Elisa Mele, ${ }^{*}$ Francesca Di Benedetto, Luana Persano, Roberto Cingolani, and \\ Dario Pisignano
}

NNL, National Nanotechnology Laboratory of INFM-CNR, clo Distretto Tecnologico

ISUFI, Università di Lecce, via Arnesano, I-73100 Lecce, Italy

Received June 28, 2005; Revised Manuscript Received August 26, 2005

\begin{abstract}
We demonstrate the multilevel patterning of organic light-emitting polymers by room-temperature nanoimprint lithography (RT-NIL), which is impossible to obtain by conventional hot embossing. In particular, we realize one- and two-dimensional photonic crystals with $500 \mathrm{~nm}$ periodic features and investigate the changes in the optical properties (luminescence and quantum yield) of the organic active layer. An increase of the quantum yield by $2.4 \%$ for the patterned film with respect to the untextured one and the enhancement of the output light emitted at a particular angle $\left(\Theta=69^{\circ}\right)$ are observed for gratings whose Bragg periodicity matched the emission wavelength of the polymer. The employment of RT-NIL to pattern polymer semiconductors without degradation of their optical properties represents a strategic route for the realization of novel nanopatterned optoelectronic devices.
\end{abstract}

Nanoimprint lithography (NIL) is a high throughput technique for producing features with size down to the sub-10 nm scale onto a polymeric layer over a large area, exploiting the glass transition phenomenology of organic materials. ${ }^{1,2}$ Differently from lithographic techniques based on the modification of the chemical structure of a resist by the use of energetic beams, the operation principle of NIL is the deformation of the surface morphology of a thermoplastic film under the application of pressure and temperature. The simple patterning procedure, whose high resolution is not limited by wave diffraction, and the low cost of the experimental setup, which does not require energetic beam generators, are the main advantages offered by NIL in comparison to the conventional lithographic approaches. In addition, this technique can be applied to a wide range of materials, both inert polymers ${ }^{1,2}$ and active compounds, ${ }^{3,4}$ which allows the realization of organic-based optoelectronic devices.

In the typical NIL process, a rigid template with a nanostructured surface is mechanically pressed onto a film of a thermoplastic polymer coating a substrate. Upon applying the pressure (in the range of $10^{5}-10^{8} \mathrm{~Pa}$ ), the polymer is heated above its glass transition temperature $\left(T_{\mathrm{g}}\right)$, thus becoming fluid and, hence, flowing into the recessed features of the stamp. After the sample is cured below $T_{\mathrm{g}}$ and the mold is separated from the polymer, a negative copy of the initial template is transferred to the target.

Because of the high values of temperature reached during the imprinting process (up to $200{ }^{\circ} \mathrm{C}$ ), ${ }^{1}$ the application of

* Corresponding author. Electronic mail: elisa.mele@unile.it.
NIL for the direct patterning of organic active molecules requires the development of a setup providing vacuum or nitrogen environments. ${ }^{4}$ In fact, the oxygen incorporation and substitution into conjugated polymers that occur at high temperature can determine the irreversible degradation of the optical and the electrical properties of the active organics. ${ }^{5}$ Nevertheless, the use of chambers with a controlled atmosphere implies both the reduction of the operational simplicity and the increase of the overall cost of NIL. Recently, roomtemperature nanoimprint lithography (RT-NIL) has been proposed as an alternative to conventional NIL for patterning thermoplastic polymers, to avoid the drawbacks of hot embossing. Since avoiding any thermal cycle, the RT-NIL can be applied directly on light-emitting molecules, in air, without degradation of the emission properties. ${ }^{3}$ The RTNIL also allows an easier mold release, and it avoids many distortions of the imprinted structures, which are possible by high-temperature imprinting. ${ }^{6}$ Importantly, RT-NIL allows one to repeat sequentially the imprinting step on the same film, to realize more complex patterns by printing the same region of the substrate with the same template for several times. In particular, we point out that the realization of twodimensional (2D) photonic crystals (PhCs) by electron-beam lithography is many times more time-consuming than that of $1 \mathrm{D}$ gratings; hence the possibility of achieving 2D nanostructures by sequential imprinting, not possible by conventional NIL, can actually be strategic for the realization of organic-based nanopatterned photonics, and particularly 2D PhCs. 
However, besides oligomer compounds, ${ }^{3}$ RT-NIL was early applied only to optically inert polymers. ${ }^{6}$ In particular, there is one important, strategic class of active materials, namely, conjugated polymers that exhibit excellent performances of luminescence ${ }^{7,8}$ and lasing, ${ }^{9,10}$ thus being ideal building blocks of micro- ${ }^{11,12}$ and nanopatterned ${ }^{13,14}$ lightemitting devices. Hence, the function-preserving, roomtemperature, possibly multilevel patterning of these compounds certainly deserves to be investigated in depth.

In this paper, we apply RT-NIL to a number of lightemitting, conjugated polymers for the realization of $1 \mathrm{D}$ and 2D periodic gratings with $500 \mathrm{~nm}$ features (by sequentially imprinting), suitable for the realization of $\mathrm{PhCs}$, and distributed feedback optical devices. ${ }^{15}$ We investigate the changes in the optical properties (luminescence and quantum yield) of the conjugated polymers, and analyze the impact of the fabricated Bragg structures on the light emission, discarding any degradation due to the imprinting process.

As for the master, we fabricated a quartz grating of period $1 \mu \mathrm{m}$ by electron-beam lithography. The geometry of the master was replicated by a negative tone photoresist (SU-8 2050), to preserve the initial structure from the damages that may occur during the imprinting process. To realize the SU-8 mold, we employed a simple and low-cost replication technology based on the use of elastomeric replicas by hard poly(dimethylsiloxane) ( $h$-PDMS). ${ }^{16-19}$ The high Young modulus $(9 \mathrm{MPa})$ of $h$-PDMS guarantees the accurate generation of high aspect ratio and nanometer scale patterns with high fidelity, avoiding mechanical collapse and pairing of the features. The pattern of the soft template was then replicated by SU-8 2050 photoresist. The SU-8 mold maintains the same shape (positive) of the starting master and can undergo the application of external pressure. It was then employed as the mold for the RT-NIL on several conjugated compounds, including a red- (RE), a green- (GE), and a blue-emitting (BE) polymer. The resist template (without any antisticking layer) was manually placed in contact with a quartz substrate spin-coated with the organic films. The system (mold and sample) was positioned on the lower plate of a precision manual press, and the imprinting was then performed in air, without heating. The applied pressure $\left(1-2 \mathrm{kN} / \mathrm{cm}^{2}\right)$ allowed us to obtain a complete pattern transfer, without forming cracks in the quartz substrate.

In Figure 1, we display the generation process of one- and two-dimensional gratings by multilevel RT-NIL. In our experiments, the $2 \mathrm{D}$ structure was realized by carrying out a second imprinting over the 1D grating, after rotating the SU-8 template $90^{\circ}$ in order to position the features of the mold perpendicular to the first patterned region. Upon removal of the stamp, a 2D pattern with $500 \mathrm{~nm}$ side squares was obtained. The possible realization of crossed patterns by multilevel imprinting is a main advantage of RT-NIL with respect to hot embossing. In fact, the heating of the target polymer film, necessary for subsequent imprinting processes by conventional NIL, would destroy the previously embossed features. On the contrary, in RT-NIL the imprinted features
I. Grating after the first imprint process

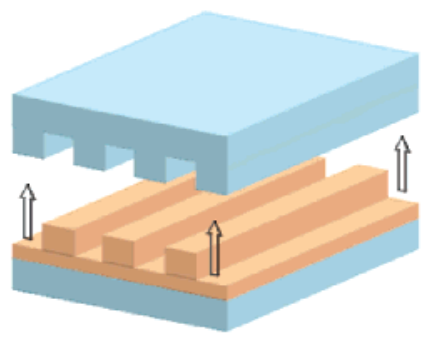

II. Second imprint process

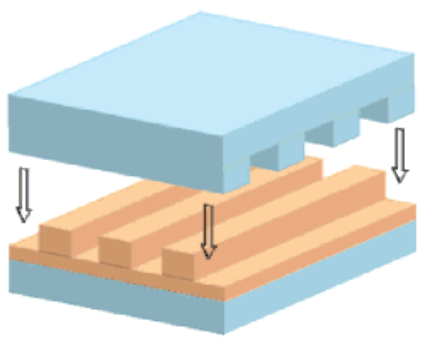

III. 2D photonic crystal

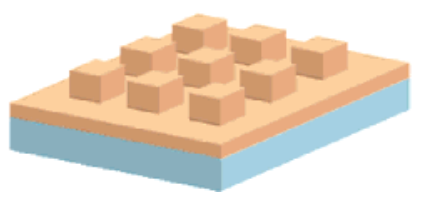

Figure 1. Schematic diagram of the RT-NIL process for the realization of $1 \mathrm{D}$ and 2D PhC-like patterns (features not in scale).

result from the irreversible physical deformation of the target layer, due to the applied pressure only.

The mechanical response of the amorphous polymers to an external stress can be explained by their viscoelastic behavior, in terms of the large conformational flexibility of the single polymer chains and the formation of a topological network of entangled chains. ${ }^{20}$ The conformational flexibility is related to the local motions of the polymeric material, whereas the weakening of the interactions between the macromolecules (entanglements) is responsible of the longrange motion of the entire chains. Upon increase of the temperature in conventional NIL, the polymer reaches its rubber-elastic region (characterized by a plateau in the curve of the time-dependent shear compliance, $\left.J(t)^{21}\right)$, where the extension of the chain segments fixed between entanglements points occurs and the deformation is reversible. At higher temperature, of for longer heating times, irreversible flow of molten material occurs (the so-called terminal flow region). In this region, the deformation induced by the external force is irreversible, and $J(t)$ exhibits a linear dependence on time. The RT imprinting intervals are quite longer than those typically employed for hot embossing, thus allowing polymers to reach their terminal-flow region and irreversible pattern transfer to be accomplished.

Moreover, in RT-NIL, an important role is also played by the contraction of the free volume, $v_{\mathrm{f}}$, in the organic film upon pressing, defined as: $v_{\mathrm{f}}=v-v_{0}$, where $v$ is the specific molecular volume and $\nu_{0}$ is the characteristic incompressible volume (hard core) of the molecule. The contraction of $v_{\mathrm{f}}$ by the application of an external stress 


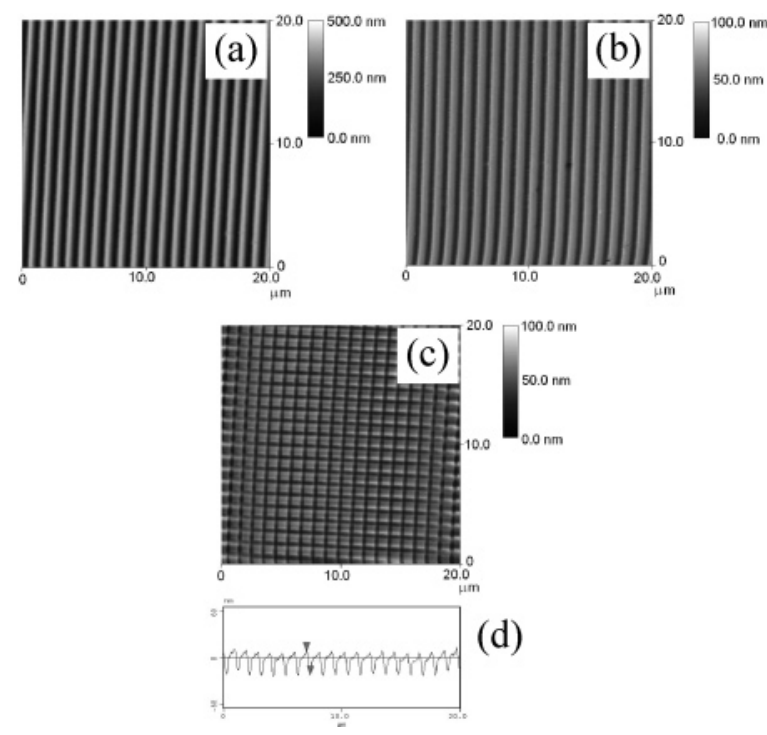

Figure 2. AFM planar view $\left(20 \times 20 \mu \mathrm{m}^{2}\right)$ of the $1 \mu \mathrm{m}$ periodic quartz master (a) and of the polymer semiconductor 1D (b) and 2D (c) imprinted gratings. (d) AFM cross section of the 2D imprinted grating.

favors the deformation of the profile of the organic film during the imprinting and the consequent conformation to the template morphology. ${ }^{6}$

The planar views of the $1 \mu \mathrm{m}$ period master, the $1 \mathrm{D}$ and 2D imprinted organic grating, imagined by atomic force microscopy (AFM), and the cross section of the 2D pattern are shown in parts a-d of Figure 2, respectively. The RTNIL allows the realization of polymeric patterns of excellent quality, both in one- and in two-dimensions. For instance, the $1 \mathrm{D}$ grating faithfully reproduces the periodicity of the starting master pattern, exhibiting a feature height of 44.0 $\pm 0.6 \mathrm{~nm}$. The displayed 2D imprinted features have a height of $28.7 \pm 1.1 \mathrm{~nm}$. Features of larger aspect ratio can be realized by increasing the duration of the imprinting process, thus providing ample time for the polymer flow under the applied pressure. The effect of the presence of 1D and 2D periodic modulations on the diffraction of light is clearly visible by eye, upon illuminating the gratings by a polychromatic source (Figure 3).

To assess the possible influence of RT-NIL on the optical properties of the light-emitting polymers, we measured the photoluminescence (PL) spectra before and after the imprinting process, by exciting the samples with a $\mathrm{HeCd}$ laser $(\lambda=$ $325 \mathrm{~nm}$ ). For all the employed compounds, the integrated PL spectra did not show variations both in the line width (full width at half-maximum, fwhm) and in the emission peak wavelength, $\lambda_{\mathrm{PL}}(646,523$, and $450 \mathrm{~nm}$ for the RE, GE, and $\mathrm{BE}$ polymer, respectively, Figure $4 \mathrm{a})$. Hence, the structuring of active materials by RT-NIL does not induce degradation of the luminescence spectral properties.

In addition, we analyzed the effects of the superimposed periodic nanostructure on the absolute quantum PL efficiency, $\eta_{\mathrm{PL}}$ (exciting the samples by a Xe lamp at $\lambda=390$ $\mathrm{nm})$. In the case of the RE compound, we found clear increase of $\eta_{\text {PL }}$ from $37.1 \%$ to $39.5 \%$ for the patterned film with respect to the untextured one. Such enhancement of
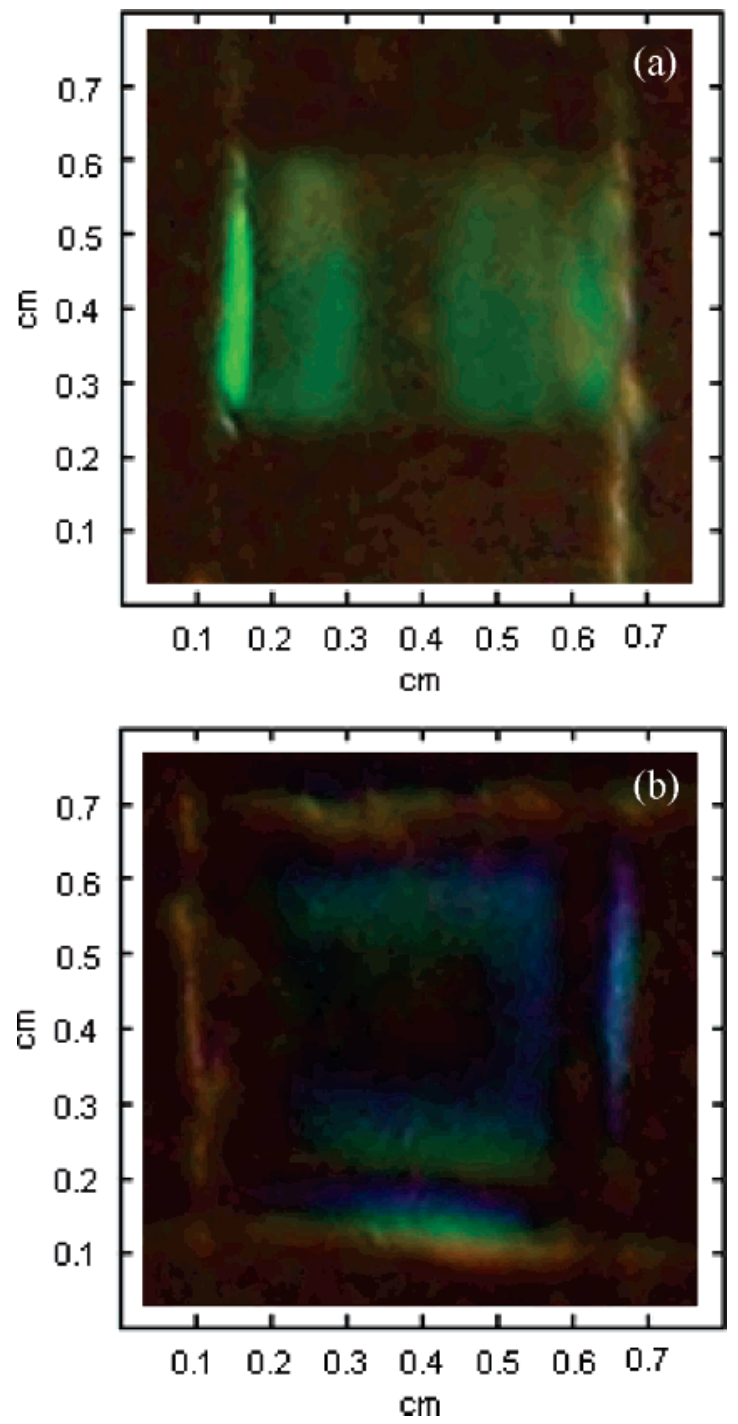

Figure 3. Photograph of the imprinted structures: (a) one and (b) two dimensional.

the PL efficiency can be attributed to the variation of the angular distribution of the light emission, as a result of the imprinted periodic corrugation. ${ }^{14}$ Indeed, for the untextured film the emitted light is preferentially waveguided in the slab, because of the higher refractive index with respect to the surrounding layers (i.e., glass and air). This decreases the resulting external luminescence efficiency as a consequence of the long paths involved in the self-absorption. Instead, the insertion of a $1 \mathrm{D}$, wavelength-scale periodic grating onto the organic film allows the light trapped in waveguide modes within the organic slab to be scattered out of the film along certain forward directions. Such enhanced Bragg scattering of light reduces the effective length covered by the photons and, consequently, the self-absorption inside the organic layer. In particular, in linear distributed feedback microcavities, ${ }^{22}$ the emission angle, $\Theta$, of the output wavelength is related to the geometry of the cavity by the relation

$$
\frac{2 \pi}{\lambda} \sin \Theta= \pm \frac{2 \pi n_{\text {eff }}}{\lambda} \pm m \frac{2 \pi}{\Lambda}
$$

where $\lambda$ is the output wavelength, $n_{\text {eff }}$ is the effective 

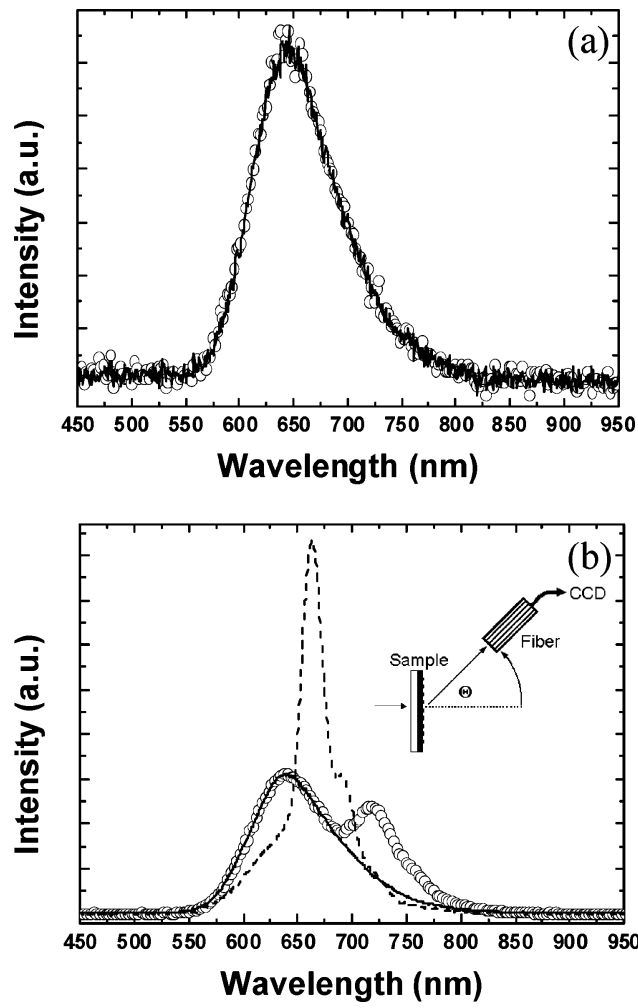

Figure 4. (a) Integrated PL spectra for the RE film before (continuous line) and after (empty circles) the RT-NIL process. (b) PL spectra from the active imprinted grating at $\Theta=0^{\circ}$ (continuous line), $59^{\circ}$ (empty circles), and $69^{\circ}$ (dotted line). Inset: experimental setup.

refractive index of the guided mode, $\Lambda$ is the period of the nanostructure, and $m$ indicates the diffraction order inside the slab. Equation 1 states the conservation of the in-plane component of the wave vector of the output light, $k_{0}=$ $\pm(2 \pi / \lambda)$, and of the guided mode $k_{\mathrm{g}}= \pm\left(2 \pi n_{\mathrm{eff}} / \lambda\right)$ by the Bragg vector (PhC reciprocal lattice vector), $G=(2 \pi / \Lambda)$. The presence of an engraved 1D PhC structure therefore determines the coupling of the guided photons to the free radiation and the increase of the forward luminance.

Consistently, in the case of the GE and BE conjugated polymers, no significant changes in $\eta_{\mathrm{PL}}$ were observed between the imprinted and the untextured films (47 and 75\% for the GE and the BE compounds, respectively). In fact, for the GE and BE polymers, the period of our grating does not match the native PL of the conjugated polymers. Thus, the printed surface modulation is unable to enhance the Bragg scattering of the emitted light out of the polymer slab.

Finally, to get a deeper insight into the angular distribution of the luminescence, we measured the PL emission at different angles, $\Theta$. The PL spectra of the patterned RE film at $0^{\circ}, 59^{\circ}$, and $69^{\circ}$ are shown in Figure 4b. The forward emission peak of the printed distributed feedback microcavity is clearly visible, shifting from $736 \mathrm{~nm}\left(\right.$ at $55^{\circ}$ ) to $649 \mathrm{~nm}$ (at $73^{\circ}$ ), with a strongly enhanced peak at $663 \mathrm{~nm}$ (at $69^{\circ}$ ). In addition, an important reduction of the fwhm from 91.0 to $26.6 \mathrm{~nm}$ can be observed. The diffraction peak depends on the collection angle, as the detection is moved perpendicularly to the direction of the patterned grooves, accord-

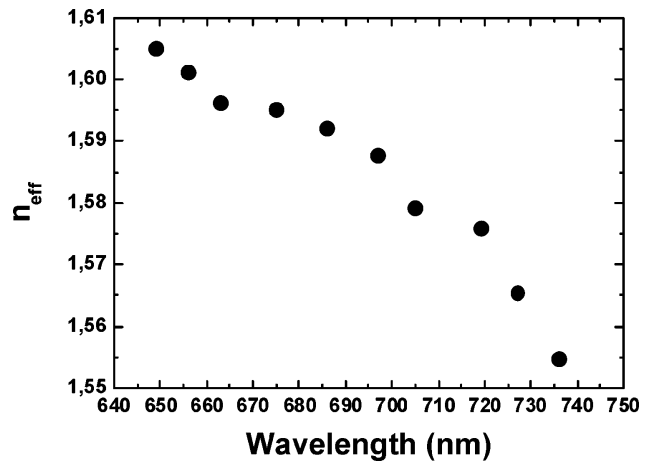

Figure 5. Wavelength dependence of $n_{\text {eff }}$ for the printed distributed feedback microcavity (RE polymer).

ingly to eq 1 . This allowed us to estimate the wavelength dependence of the effective refractive index, $n_{\text {eff }}$, inside the organic slab, getting values between 1.61 and 1.55 for wavelengths between 640 and $740 \mathrm{~nm}$ (Figure 5).

In conclusion, we demonstrated that RT-NIL enables the multilevel patterning of organic light-emitting polymers, impossible to obtain by conventional hot embossing. These results therefore extend the field of application of roomtemperature imprinting on active molecular materials from low-molar-mass ${ }^{3}$ compounds to conjugated polymers. In particular, we realized high-quality $1 \mathrm{D}$ and 2D PhCs with $500 \mathrm{~nm}$ periodic features and analyzed the effect of imprinting the periodic corrugation onto the organic active layer. An increase of the quantum yield by $2.4 \%$ for the patterned film with respect to the untextured one and the enhancement of the output light emitted at a particular angle $\left(\Theta=69^{\circ}\right)$ were observed, for gratings whose Bragg periodicity matched the emission wavelength of the polymer. The employment of RT-NIL to pattern polymer semiconductors without degradation of their optical properties represents a strategic route for the realization of novel nanopatterned optoelectronic devices.

Supporting Information Available: Descriptions of the materials, procedures, and instrumentation used. This material is available free of charge via the Internet at http:// pubs.acs.org.

\section{References}

(1) (a) Chou, S. Y.; Krauss, P. R.; Zhang, W.; Guo, L.; Zhuang, L. $J$. Vac. Sci. Technol., B 1997, 15, 2897-2904. (b) Chou, S. Y.; Krauss, P. R.; Renstrom, P. J. Science 1996, 272, 85-87.

(2) Heidari, B.; Maximov, I.; Montelius, L. J. Vac. Sci. Technol., B 2000 $18,3557-3560$.

(3) Pisignano, D.; Persano, L.; Raganato, M. F.; Visconti, P.; Cingolani, R.; Barbarella, G.; Favaretto, L.; Gigli, G. Adv. Mater. 2004, 16, 525-529.

(4) Wang, J.; Sun, X.; Chen, L.; Chou, S. Y. Appl. Phys. Lett. 1999, 75, $2767-2769$.

(5) (a) Yan, M.; Rothberg, L. J.; Papadimitrakopoulos, F.; Galvin, M. E.; Miller, T. M. Phys. Rev. Lett. 1994, 73, 744-747. (b) Papadimitrakopoulos, F.; Konstadinidis, K.; Miller, T. M.; Opila, R.; Chandross, E. A.; Galvin, M. E. Chem. Mater. 1994, 6, 1563-1568.

(6) Khang, D.-Y.; Yoon, H.; Lee, H. H. Adv. Mater. 2001, 13, 749752.

(7) Friend, R. H.; Gymer, R. W.; Holmes, A. B.; Burroughes, J. H.; Marks, R. N.; Taliani, C.; Bradley, D. D. C.; Dos Santos, D. A.; Brédas, J. L.; Lögdlund, M.; Salaneck, W. R. Nature 1999, 397, 121128. 
(8) Kraft, A.; Grimsdale, A. C.; Holmes, A. B. Angew. Chem., Int. Ed. 1998, 37, 402-428.

(9) Hide, F.; Díaz-García, M. A.; Schwartz, B. J.; Andersson, M. R.; Pei, Q.; Heeger, A. J. Science 1996, 273, 1833-1836.

(10) Friend, R. H.; Denton, G. J.; Tessler, N.; Stevens, M. A. Adv. Mater. 1997, 9, 547-551.

(11) Xia, R.; Heliotis, G.; Bradley, D. D. C. Appl. Phys. Lett. 2003, 82, 3599-3601.

(12) Birnstock, J.; Blässing, J.; Hunze, A.; Scheffel, M.; Stössel, M.; Heuser, K.; Wittmann, G.; Wörle, J.; Winnacker, A. Appl. Phys. Lett. 2001, 78, 3905-3907.

(13) Müller, C. D.; Falcou, A.; Reckefuss, N.; Rojahn, M.; Wiederhirn, V.; Rudati, P.; Frohne, H.; Nuyken, O.; Becker, H.; Meerholz, K. Nature 2003, 421, 829-833.

(14) (a) Matterson, B. J.; Lupton, J. M.; Safonov, A. F.; Salt, M. G.; Barnes, W. L.; Samuel, I. D. W. Adv. Mater. 2001, 13, 123-127. (b) Ziebarth, J. M.; Saafir, A. K.; Fan, S.; McGehee, M. D. Adv. Funct. Mater. 2004, 14, 451-456.
(15) Boroumand, F. A.; Fry, P. W.; Lidzey, D. G. Nano Lett. 2005, 5, $67-71$

(16) Kogelnik, H.; Shank, C. V. Appl. Phys. Lett. 1971, 18, 152154.

(17) Xia, Y.; Whitesides, G. M. Angew. Chem., Int. Ed. 1998, 37, 550575.

(18) Xia, Y.; Kim, E.; Zhao, X.-M.; Rogers, J. A.; Prentiss, M.; Whitesides, G. M. Science 1996, 273, 347-349.

(19) Schmid, H.; Michel, B. Macromolecules 2000, 33, 3042-3049.

(20) Zankovych, S.; Hoffmann, T.; Seekamp, J.; Bruch, J.-U.; Sotomayor Torres, C. M. Nanotechnology 2001, 12, 91-95.

(21) Strobl, G. The Physics of Polymers; Springer: Berlin, 1997.

(22) Turnbull, G. A.; Andrew, P.; Jory, M. J.; Barnes, W. L.; Samuel, I. D. W. Phys. Rev. B 2001, 64, 125122.

NL051234P 